\title{
Magnetically Assisted Fast Ignition
}

\author{
W.-M. Wang, ${ }^{1,2,5,{ }^{*}}$ P. Gibbon, ${ }^{1}$ Z.-M. Sheng, ${ }^{3,4,5}$ and Y.-T. Li ${ }^{2,5}$ \\ ${ }^{1}$ Forschungszentrum Jülich GmbH, Institute for Advanced Simulation, Jülich Supercomputing Centre, D-52425 Jülich, Germany \\ ${ }^{2}$ Beijing National Laboratory for Condensed Matter Physics, Institute of Physics, CAS, Beijing 100190, China \\ ${ }^{3}$ SUPA, Department of Physics, University of Strathclyde, Glasgow G4 ONG, United Kingdom \\ ${ }^{4}$ Key Laboratory for Laser Plasmas (MoE) and Department of Physics and Astronomy, \\ Shanghai Jiao Tong University, Shanghai 200240, China \\ ${ }^{5}$ IFSA Collaborative Innovation Center, Shanghai Jiao Tong University, Shanghai 200240, China
}

(Received 1 October 2014; published 7 January 2015)

\begin{abstract}
Fast ignition (FI) is investigated via integrated particle-in-cell simulation including both generation and transport of fast electrons, where petawatt ignition lasers of $2 \mathrm{ps}$ and compressed targets of a peak density of $300 \mathrm{~g} \mathrm{~cm}^{-3}$ and areal density of $0.49 \mathrm{~g} \mathrm{~cm}^{-2}$ at the core are taken. When a $20 \mathrm{MG}$ static magnetic field is imposed across a conventional cone-free target, the energy coupling from the laser to the core is enhanced by sevenfold and reaches $14 \%$. This value even exceeds that obtained using a cone-inserted target, suggesting that the magnetically assisted scheme may be a viable alternative for FI. With this scheme, it is demonstrated that two counterpropagating, $6 \mathrm{ps}, 6 \mathrm{~kJ}$ lasers along the magnetic field transfer $12 \%$ of their energy to the core, which is then heated to $3 \mathrm{keV}$.
\end{abstract}

DOI: 10.1103/PhysRevLett.114.015001

The fast ignition (FI) scheme in laser-driven intertial confinement fusion has attracted much attention because of its potential for reducing the driver energy requirements, but its realization has revealed significant technical challenges since it was proposed 20 years ago [1]. FI requires that a large quantity of collimated fast electrons of $\mathrm{MeV}$ are transported over a $100 \mu \mathrm{m}$ distance in coronal plasma to heat a high-density core at $300 \mathrm{~g} \mathrm{~cm}^{-3}$, where the fast electrons are generated by a 10 ps petawatt $(\mathrm{PW})$ ignition laser. A key outstanding issue is how to achieve a reasonable coupling of $10 \%$ or more from the laser to the core. Up to $20 \%$ coupling from a 0.6 ps ignition laser was demonstrated experimentally in 2001 [2] with a coneinserted target to reduce the transport distance of the electrons to the core. However, a few subsequent experiments with longer duration ignition lasers between 2008 and 2011 reported much lower coupling at Vulcan [3], Omega EP [4], and GEKKO XII systems [5], respectively. The large difference in the coupling was not completely understood, but could be related to different preplasmas formed by the ignition laser prepulses in the cones [3-5]. A commonly cited factor causing the low coupling in these experiments [3-5] is large divergence of the fast electrons generated in the cones [6,7]. Divergence angles up to $50^{\circ}$ were found in many studies [7-9].

In this Letter, we propose a new route for higher coupling based upon integrated particle-in-cell (PIC) simulation using a recently developed code [10], where fast electron generation, electron transport, and energy deposition in a compressed target are all included explicitly. This provides a straightforward way to compare the laser-to-core coupling among different schemes quantitatively. Here we propose to use a cone-free target supplemented by an
PACS numbers: 52.57.Kk, 52.38.-r, 52.65.Rr, 52.65.Ww

external, static magnetic field ( $B$ field). Such a spherically symmetric target does not suffer from asymmetry in target compression and subsequent reduction in the areal density of the compressed target [11], as is the case for a coneinserted target [2]. The applied $B$ field confines the fast electron motion and reduces the impact of the large initial divergence. The divergence is expected to be even more detrimental with a cone-free target than a cone-inserted target, since a longer distance is needed to transport the fast electrons. Even though previous work indicates that such a $B$ field may help to overcome the fast electron divergences $[7,12]$, a quantitative assessment of how much the $B$ field can improve the coupling is still lacking. In this Letter, we show that the $B$ field with strength $20 \mathrm{MG}$ applied along the ignition laser propagation direction can enhance laser-to-core coupling by sevenfold, exceeding the efficiency obtained with the cone-inserted scheme. Note that such $B$ fields have recently been generated in laser-driven magnetic-flux compression experiments [13] and nanosecond-laser-driven capacitor-coil experiments [14].

We first take 2 ps ignition lasers to compare the coupling among the original, cone-inserted, and magnetically assisted (MA) schemes. The simulations are implemented by the two-dimensional (2D) KLAPS with a two-system PIC model developed recently [10]. Fast electron generation via laser-plasma interaction is simulated by a conventional PIC system. When the fast electrons transport to the region with the plasma density $200 n_{c}\left(n_{c}=1.1 \times 10^{21} \mathrm{~cm}^{-3}\right)$ or to the cone tip in the cone-inserted scheme, where it is far away from the laser interaction zone, the data of these fast electrons are copied in real time to a second PIC system utilizing a reduced field solver as in previously reported two-region [15] and hybrid [16] PIC methods. We define 
the fast electrons as having energy above $0.1 \mathrm{MeV}$ and forward momentum $p_{x}>0.45 m_{e} c(50 \mathrm{keV})$. The second PIC system calculates the subsequent transport of these electrons in real target density (with a pedestal of $198 n_{c}$ ), as shown in Figs. 1(a)-1(c). In both systems macroparticles are taken to denote the plasma and Coulomb collision [17] and 4th-order current calculation [10] is included. In the conventional system, if the density is above $200 n_{c}$, it is lessened artificially to this value to reduce numerical noise [10].

For simplicity we take tritium targets instead of deuterium-tritium targets since fusion processes are not considered here. The targets in the three schemes are shown in Figs. 1(a)-1(c), with a uniform density of $300 \mathrm{~g} \mathrm{~cm}^{-3}$ $\left(54000 n_{c}\right)$ within a circle of radius $10 \mu \mathrm{m}$ and the surrounding density decreasing exponentially with scale length $9 \mu \mathrm{m}$ along the radial direction. The critical density layer is located $108 \mu \mathrm{m}$ away from the target center. We define the area above $100 \mathrm{~g} \mathrm{~cm}^{-3}$ as the core with areal density $0.49 \mathrm{~g} \mathrm{~cm}^{-2}$. Implosion simulations [18] show the plasma temperature is within $0.3-1 \mathrm{keV}$. For simplicity we employ an uniform temperature of $1 \mathrm{keV}$ for electrons and ions. In Fig. 1(a) a cone is used with wall depth $5 \mu \mathrm{m}$, density $200 n_{c}$, tip size $20 \mu \mathrm{m}$, and inner length $20 \mu \mathrm{m}$. The cone opening angle of $30^{\circ}$ and the distance of $35 \mu \mathrm{m}$ between the tip to target center approach the experimental parameters [3-5]. To allow for prepulse effects, a preplasma is taken inside the cone with an exponential profile with scale length $2 \mu \mathrm{m}$ along the $x$ direction (our simulation shows the laser-to-core coupling is reduced by $25 \%$ with the increased scale length $4 \mu \mathrm{m}$, and the coupling approaches the experimental result in [4]). A $0.63 \mathrm{~kJ}$ ignition laser propagates along the $+x$ direction with wavelength $1 \mu \mathrm{m}$ and electric field $E_{y}=$ $a_{0} \exp \left(-y^{2} / r_{0}^{2}\right) f(\xi) \sin (2 \pi \xi)$, where $a_{0}=12.1$ corresponding to $2 \times 10^{20} \mathrm{~W} / \mathrm{cm}^{2}, \xi=t-x / c, r_{0}=10 \mu \mathrm{m}$; the temporal profile $f(\xi)$ is taken as an infinite plateau after 33 fs rising edge. The simulation box size $176 \mu \mathrm{m} \times$ $128 \mu \mathrm{m}(128 \mu \mathrm{m} \times 128 \mu \mathrm{m}$ in the cone-inserted case $)$ in the $x \times y$ directions is taken in the two PIC systems. The spatial resolution is $0.02 \mu \mathrm{m}$. In the conventional system 49 electrons and ions per cell are taken to control the noise, 25 in the second system.

Figures 1(d)-1(f) show fast-electron currents with the three schemes in the second PIC system. With the original scheme the fast electrons diffuse in the whole transverse space in the $y$ direction [see Fig. 1(e)] due to large divergence. With a cone inserted, a strengthened current is distributed in reduced transverse space [Fig. 1(d)]. Most strikingly, when a $20 \mathrm{MG}$ static $B$ field is applied to the cone-free target along the $x$ direction [Fig. 1(c)], the fastelectron current is confined around the axis within a narrower transverse space [Fig. 1(f)] than the cone-inserted scheme. The current in Fig. 1(f) is weaker than that in Fig. 1(d) because the former is distributed in a larger longitudinal space and composed of fewer electrons with higher energy, as discussed below. Resistive electric fields consistent with the currents are plotted in Figs. 1(g)-1(i),
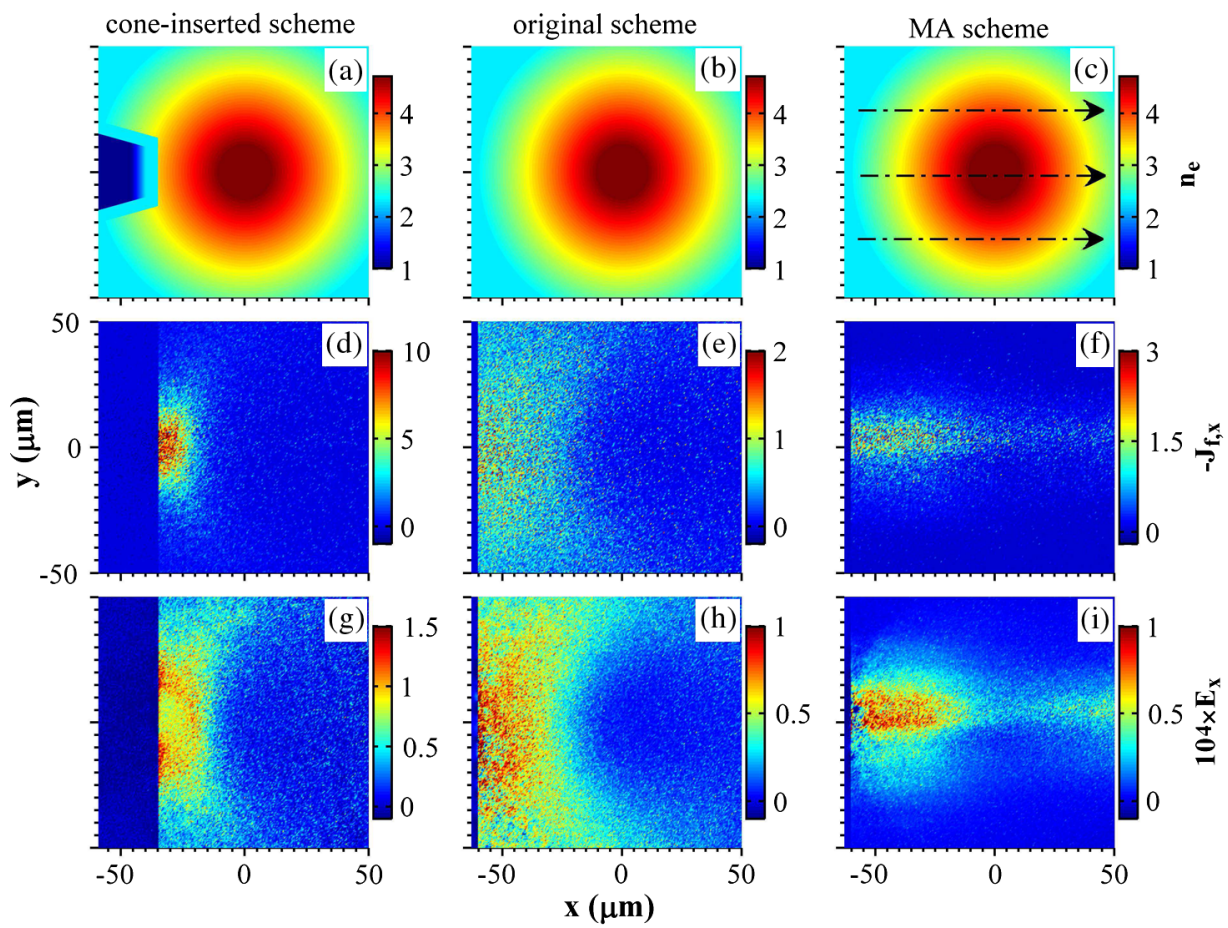

FIG. 1 (color online). Snapshots of electron densities $\lg \left(n_{e} / n_{c}\right)$ at initial time (first row), fast-electron currents $J_{f, x} / e n_{c} c$ at 2 ps (second row), and resistive electric fields $10^{4} \times e E_{x} / m_{e} \omega c$ at 2 ps (third row). The three columns correspond to the cone-inserted, original, and MA schemes, respectively. 


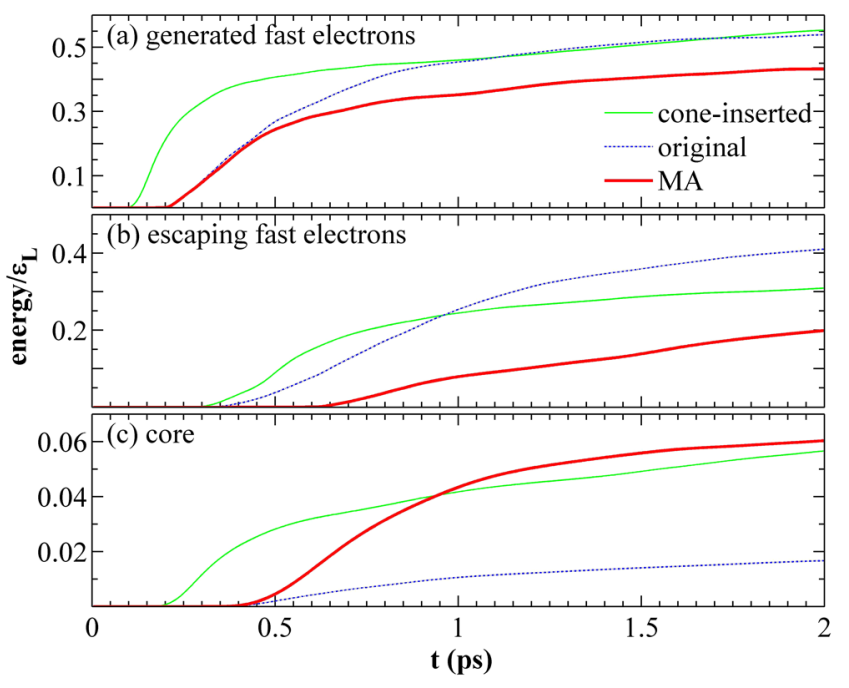

FIG. 2 (color online). Temporal evolution of fractional energy of the fast electrons (a) generated from laser interaction, (b) escaping from the simulation box, and (c) absorbed by the core, normalized by the laser energy $\varepsilon_{L}$. Different lines in each plot correspond to the cone-inserted, original, and MA schemes, respectively.

which have similar patterns to the currents. In Fig. 1(i) the field vanishes at the region far away from the axis $(y=0)$, in contrast to Fig. 1(g). This suggests fewer electrons escape transversely away from the simulation box in the MA scheme. By contrast, more electrons escape longitudinally after they travel through the target center, according to the field distributed at $x>0$ in Fig. 1(i).

Figure 2(b) displays the energy of the escaping fast electrons with time. In the MA scheme the escaping electron fraction is reduced considerably compared with the other two schemes. Whereas, this does not bring much advantage in the laser-to-core coupling shown in Fig. 2(c): $6.2 \%$ for the MA scheme at 2 ps and $5.6 \%$ for the coneinserted scheme. There are two main reasons for this. First, the intensity $2 \times 10^{20} \mathrm{~W} / \mathrm{cm}^{2}$ used is too high for the conefree target, in which the average energy of the fast electrons generated is $4.8 \mathrm{MeV}$ (counted at $x=-60 \mu \mathrm{m}$, where fast electrons are injected to the second system). This energy is far above $1.2 \mathrm{MeV}$ (counted at the injection point $x=-35 \mu \mathrm{m})$ in the cone-inserted case, due to the larger density scale length $9 \mu \mathrm{m}$ in the MA scheme compared to the preplasma density scale length $2 \mu \mathrm{m}$ in the cone [19]. Therefore, the fast electrons escape mainly longitudinally in the MA scheme, as shown in Table I. In fact, this laser intensity has been optimized for the cone-inserted scheme according to Ref. [20]. The authors found that the laser-tocore coupling is nearly unchanged with growing intensity at relatively low values and falls at higher intensities. A similar result is shown in Table I with an optimal intensity at $2 \times 10^{20} \mathrm{~W} / \mathrm{cm}^{2}$. A second reason is that the coupling to the fast electrons $\left(p_{x}>0.45 m_{e} c\right)$ is reduced with the $B$ field [see Fig. 2(a) and Table I]. Our simulations
TABLE I. Energy coupling (percentage) from the laser to the core $\eta_{\text {core }}$ and to the fast electrons $\eta_{\text {fast }}$, and the laser reflectivity $R$ at different intensities $\left(\mathrm{W} / \mathrm{cm}^{2}\right)$ when original, cone-inserted, and MA schemes are taken, respectively. "Escape" is the energy of all the escaping fast electrons and Escape $_{\perp}$ is that escaping transversely. These values are obtained at $2 \mathrm{ps}$. Each simulation takes $0.8 \times 10^{6}$ core hours on average on JUQUEEN.

\begin{tabular}{lcrcccr}
\hline \hline Scheme & Intensity & $\eta_{\text {core }}$ & $\eta_{\text {fast }}$ & Escape & Escape $_{\perp}$ & \multicolumn{1}{c}{$R$} \\
\hline Original & $5 \times 10^{19}$ & 2.1 & 51.2 & 38.1 & 26.2 & 6.8 \\
Original & $2 \times 10^{20}$ & 1.6 & 53.8 & 40.9 & 28.1 & 6.2 \\
Cone-inserted & $1 \times 10^{20}$ & 5.7 & 50.6 & 27.4 & 14.5 & 27.0 \\
Cone-inserted & $2 \times 10^{20}$ & 5.6 & 55.2 & 30.8 & 15.2 & 24.2 \\
Cone-inserted & $4 \times 10^{20}$ & 4.6 & 53.8 & 33.6 & 17.1 & 20.1 \\
MA & $5 \times 10^{19}$ & 13.9 & 48.2 & 16.4 & 0.08 & 13.6 \\
MA & $1 \times 10^{20}$ & 10.0 & 48.6 & 19.3 & 0.09 & 10.4 \\
MA & $2 \times 10^{20}$ & 6.0 & 43.1 & 19.8 & 1.4 & 9.3 \\
\hline \hline
\end{tabular}

show that the $B$ field causes stronger hole boring [19] and more laser energy is reflected or scattered by the plasma with higher density. These reflected and scattered light beams can generate hot electrons largely deviating from (even opposite to) the $+x$ direction.

To optimize the laser-to-core coupling for the MA scheme, we decrease the laser intensity to reduce the electron energy. The coupling enhances continuously and reaches $13.9 \%$ at $5 \times 10^{19} \mathrm{~W} / \mathrm{cm}^{2}$ (Table I) with average electron energy $2.9 \mathrm{MeV}$. It should grow with further decrease in the intensity. Considering that PW-scale ignition lasers will be adopted in real experiments, laser intensities should not be too low. To achieve high coupling with relatively high intensities, one can employ $2 \omega$ or $3 \omega$ lasers since the electron energy scales approximately linearly with the laser wavelength $[21,22]$.

In further simulations, we take $2 \omega$ lasers at $2 \times$ $10^{20} \mathrm{~W} / \mathrm{cm}^{2}$ expecting to obtain the coupling as high as that with the fundamental laser at $5 \times 10^{19} \mathrm{~W} / \mathrm{cm}^{2}$. Two counterpropagating lasers along the $B$ field are adopted with duration $6 \mathrm{ps}$ and a slightly enhanced $r_{0}$ of $12.6 \mu \mathrm{m}$. Each laser has a power $0.5 \mathrm{PW}$ and energy $3 \mathrm{~kJ}$. The simulation box is increased to $224 \mu \mathrm{m}$ in the $x$ direction to include the second laser incidence. The target parameters are not changed. This simulation takes $3 \times 10^{6}$ core hours on JUQUEEN.

Figure 3 shows the target temperatures at different times, where the core is marked by a circle. It illustrates the process of the core heating by two counterpropagating fast-electron influxes, as shown in Fig. 4. Because the influxes are directed, the heating front always appears around the axis, which is favorable for the heating to the core. At 2 ps the core periphery starts to be heated [Fig. 3(a)]. At 6 ps the whole core has been obviously heated [Fig. 3(e)] and the average electron temperature reaches $3 \mathrm{keV}$ [Fig. 5(c)]. Note that the fast electrons are included to calculate the temperatures in Figs. 3 and 5(c). Following the electron heating, the ions are also heated as 


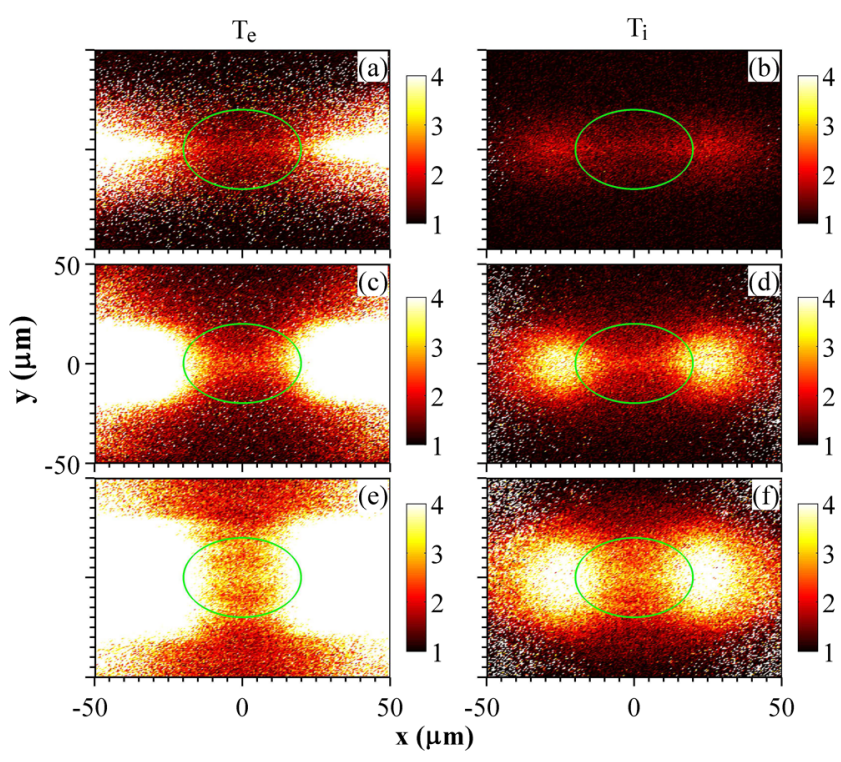

FIG. 3 (color online). Snapshots of temperatures $(\mathrm{keV})$ of electrons (left column) and ions (right column). The three rows correspond to 2, 4, and $6 \mathrm{ps}$, respectively. The core is marked with the green circle in each plot.

observed in Figs. 3(b), 3(d), and 3(f). At 6 ps the average ion temperature at the core is $2.8 \mathrm{keV}$ [Fig. 5(c)]. At this time the coupling from the two lasers to the core is $12 \%$, as shown in Fig. 5(b).

A double-lobe pattern is observed in the ion temperature distributions; i.e., the ions are preferentially heated at two symmetric regions outside the core and inside the two injection points $(x= \pm 60 \mu \mathrm{m})$. The reason is as follows. The plasma density is relatively low around the injection

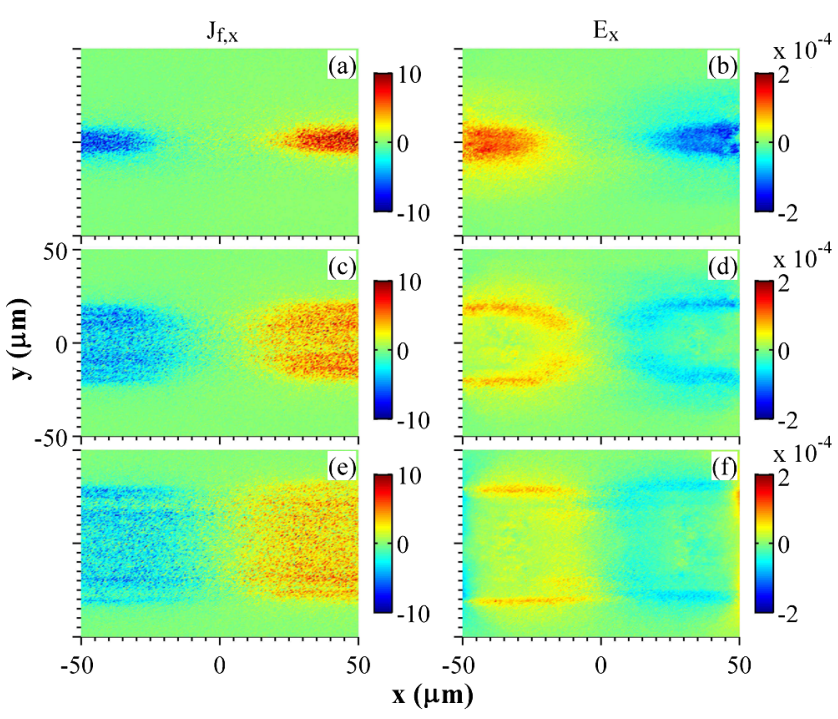

FIG. 4 (color online). Snapshots of fast-electron currents $\left(e n_{c} c\right)$ in the left column and resistive electric fields $\left(m_{e} \omega c / e\right)$ in the right column. The three rows correspond to 2,4 , and $6 \mathrm{ps,}$ respectively. points and the fast electrons have too high energy here at earlier times, as seen in Fig. 5(c). Hence, collisions between these electrons and background ions are weak. With transport of these electrons towards the target center, their energy is reduced gradually, the plasma density grows, and therefore the collisions become stronger. On the other hand, in the lower-density region the temperature enhancement shows more remarkably if the same energy is absorbed. Thus the hottest regions appear between the injection points and the target center. As the fast-electron energy decreases continuously [see Fig. 5(c)], the hottest regions spread towards the injection points as seen in Fig. 3(f). Meanwhile, the temperature around the target center shows obvious enhancement due to temporal accumulation of energy absorption.

Figure 5(c) shows that fast-electron average energy decreases with time, implying that it is not accurate to take an invariant energy spectrum of fast electrons as in most hybrid-PIC simulations (often to save computational expense). A reason is that electrons with lower energy arrive at the injection point with retardation. Another is laser hole boring. The lasers first interact with the lowerdensity plasma and generate electrons with higher energy. Then they enter into a deeper region with higher density and produce lower-energy electrons. During this process the reflectivity grows with time and after 3 ps the coupling to the fast electrons decreases, as shown in Fig. 5(a). With lower energy, these electrons heat the target more efficiently and, therefore, the coupling to the target and core does not decrease, as shown in Fig. 5(b).

Note that the fast-electron currents are computed also with the background electrons above 5 times local

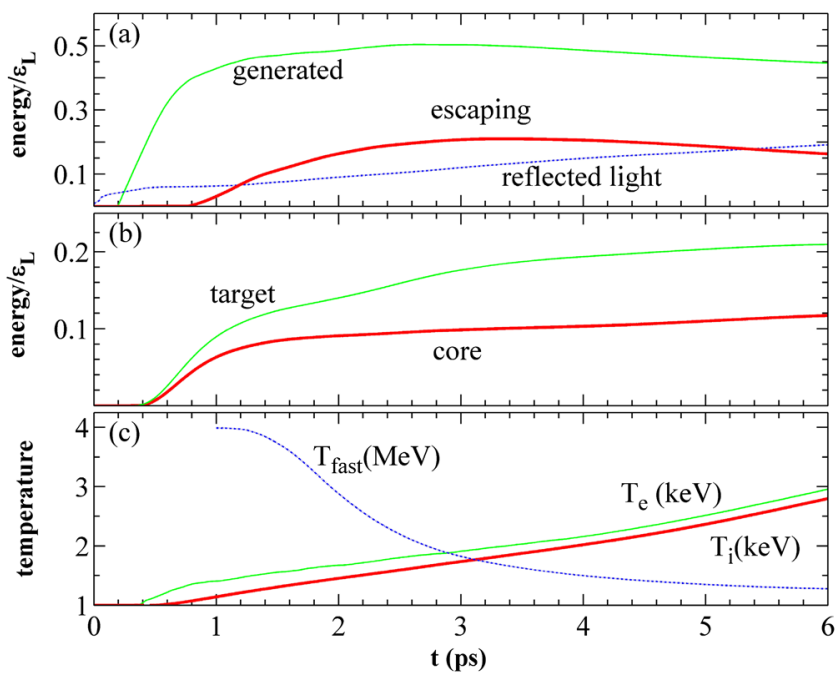

FIG. 5 (color online). (a) Temporal evolution of energy of the fast electrons generated, the escaping ones, and the reflected light. (b) Energy gained by the whole target and the core. These energies are normalized by the laser energy $\varepsilon_{L}$ entering into the simulation box. (c) Temperatures (average energy) of the core electrons and ions and the fast electrons. 
temperature. Thus the currents become wider with time in Fig. 4. One notices in Figs. 4(d) and 4(f) that the fields at peripheries are stronger because the two counterpropagating influxes with higher-energy electrons around the axis travel through the target center and counteract. Besides, our simulations show that collisions dominate completely over Ohmic heating, as observed in hybrid-PIC simulations [6]. Contribution of self-generated fields (including the azimuthal $B$ field) from the beam to the core heating is slight.

We have taken 2D simulations. This is expected to cause small difference in the laser-to-core coupling compared to 3D simulations due to the symmetry of transverse electron motion under the $B$ field; i.e., if electrons hit on the core at a 2D circle, they can reach the corresponding 3D sphere. Also, our simulations do not include implosion and therefore the evolution of the imposed $B$-field topology and the target conditions are not considered. Simply, we have used the initial $B$-field topology, which could be suitable if the $B$ field can be imposed shortly before the ignition laser incidence. Besides, we have taken the injection point at $200 n_{c}$ to meet the conditions of the two-system approach [10]: it is far away from the laser interaction zone; the density here should be sufficiently high to satisfy the field solver in the second system; the density should also be low enough to avoid the noise in the conventional system.

In summary, we have explicitly demonstrated high laserto-core coupling through a static $B$ field imposed on a conefree target with the help of large scale integrated PIC simulations. The coupling reaches $14 \%$ at a slightly optimized laser intensity, compared to $2 \%$ without the $B$ field. This is attributed to the constrained fast-electron motion along the $B$ field. The coupling with the coneinserted target is 6\% at an optimized laser intensity and without considering implosion asymmetry. We have shown that the high coupling via the MA scheme can maintain for 6 ps with $2 \omega$ ignition lasers. The coupling could be enhanced further provided $3 \omega$ lasers are taken to reduce the fast-electron energy further.

W. M. W. acknowledges support from the Alexander von Humboldt Foundation. The authors gratefully acknowledge the computing time granted by the JARA-HPC and VSR committees on the supercomputer JUQUEEN at Forschungszentrum Jülich. This work was supported by the National Basic Research Program of China (Grant No. 2013CBA01500) and NSFC (Grants No. 11375261, 11105217, 11421064, 11129503, 113111048, 11375262, and 11135012).

*weiminwang1@126.com

[1] M. Tabak, J. Hammer, M. Glinsky, W. Kruer, S. Wilks, J. Woodworth, E. Campbell, M. Perry, and R. Mason, Phys. Plasmas 1, 1626 (1994).
[2] R. Kodama, P. A. Norreys, K. Mima, A. E. Dangor, R. G. Evans, H. Fujita, Y. Kitagawa, K. Krushelnick, T. Miyakoshi, N. Miyanaga et al., Nature (London) 412, 798 (2001).

[3] M.H. Key, J. C. Adam, K. U. Akli, M. Borghesi, M. H. Chen, R. G. Evans, R. R. Freeman, H. Habara, S. P. Hatchett, J. M. Hill et al., Phys. Plasmas 15, 022701 (2008).

[4] W. Theobald, A. A. Solodov, C. Stoeckl, K. S. Anderson, R. Betti, T. R. Boehly, R. S. Craxton, J. A. Delettrez, C. Dorrer, J.A. Frenje et al., Phys. Plasmas 18, 056305 (2011).

[5] H. Shiraga, S. Fujioka, M. Nakai, T. Watari, H. Nakamura, Y. Arikawa, H. Hosoda, T. Nagai, M. Koga, H. Kikuchi et al., Plasma Phys. Controlled Fusion 53, 124029 (2011).

[6] J. Honrubia and J. Meyer-ter-Vehn, Nucl. Fusion 46, L25 (2006).

[7] D. J. Strozzi, M. Tabak, D. J. Larson, L. Divol, A. J. Kemp, C. Bellei, M. M. Marinak, and M. H. Key, Phys. Plasmas 19, 072711 (2012).

[8] R. B. Stephens, R. A. Snavely, Y. Aglitskiy, F. Amiranoff, C. Andersen, D. Batani, S. D. Baton, T. Cowan, R. R. Freeman, T. Hall et al., Phys. Rev. E 69, 066414 (2004).

[9] Z.-M. Sheng, Y. Sentoku, K. Mima, J. Zhang, W. Yu, and J. Meyer-ter-Vehn, Phys. Rev. Lett. 85, 5340 (2000).

[10] W.-M. Wang, P. Gibbon, Z.-M. Sheng, and Y.-T. Li, Phys. Rev. E 91, 013101 (2015).

[11] C. Stoeckl, T. R. Boehly, J. A. Delettrez, S. P. Hatchett, J. A. Frenje, V. Yu. Glebov, C. K. Li, J. E. Miller, R. D. Petrasso, F. H. Seguin et al., Plasma Phys. Controlled Fusion 47, B859 (2005).

[12] H.-B. Cai, S.-P. Zhu, and X. T. He, Phys. Plasmas 20, 072701 (2013).

[13] P. Y. Chang, G. Fiksel, M. Hohenberger, J. P. Knauer, R. Betti, F. J. Marshall, D. D. Meyerhofer, F. H. Seguin, and R. D. Petrasso, Phys. Rev. Lett. 107, 035006 (2011).

[14] S. Fujioka, Z. Zhang, K. Ishihara, K. Shigemori, Y. Hironaka, T. Johzaki, A. Sunahare, N. Yamamoto, H. Nakashima, T. Watanabe et al., Sci. Rep. 3, 1170 (2013).

[15] B. I. Cohen, A. J. Kemp, and L. Divol, J. Comput. Phys. 229, 4591 (2010).

[16] A. R. Bell, J. R. Davies, S. Guerin, and H. Ruhl, Plasma Phys. Controlled Fusion 39, 653 (1997).

[17] Y. Sentoku and A. J. Kemp, J. Comput. Phys. 227, 6846 (2008).

[18] J. J. Honrubia, J. Quant. Spectrosc. Radiat. Transfer 49, 491 (1993).

[19] P. Gibbon, Short Pulse Laser Interactions with Matter (Imperial College Press, London, 2000).

[20] B. Chrisman, Y. Sentoku, and A. J. Kemp, Phys. Plasmas 15, 056309 (2008).

[21] S. C. Wilks, W. L. Kruer, M. Tabak, and A. B. Langdon, Phys. Rev. Lett. 69, 1383 (1992).

[22] Y.-Q. Cui, W.-M. Wang, Z.-M. Sheng, Y.-T. Li, and J. Zhang, Plasma Phys. Controlled Fusion 55, 085008 (2013). 